Research Paper

\title{
Inhibition of Sirtuin 3 prevents titanium particle-induced bone resorption and osteoclastsogenesis via suppressing ERK and JNK signaling
}

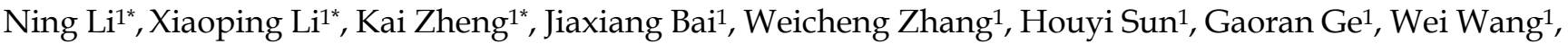 \\ Zhen Wang 2 , Ye Gu${ }^{3}$, Yi Xue ${ }^{4}$, Yaozeng $\mathrm{Xu}^{1 凶}$, Dechun Geng ${ }^{1 凶}$ and Jun Zhou ${ }^{1 凶}$ \\ 1. Department of Orthopaedics, The First Affiliated Hospital of Soochow University, No. 188 Shizi Street, Suzhou, Jiangsu 215006, China. \\ 2. Department of Orthopaedics, Suzhou Kowloon Hospital Shanghai Jiao Tong University School of Medicine, Suzhou, Jiangsu 215006, China. \\ 3. Department of Orthopedics, Soochow University Affiliated First People's, Hospital of Changshou City, Changshu, Jiangsu 215500, China. \\ 4. Department of Orthopaedics, Changshu Hospital Affiliated to Nanjing University of Traditional Chinese Medicine, Suzhou, Jiangsu 215500, China. \\ *These authors contributed equally to this work. \\ $\bowtie$ Corresponding authors: Prof. Jun Zhou. E-mail. zhou.jun.roy@hotmail.com; Prof. Dechun Geng. E-mail: szgengdc@suda.edu.cn; Prof. Yaozeng Xu. E-mail: \\ xuyaozeng@163.com.
}

(1) The author(s). This is an open access article distributed under the terms of the Creative Commons Attribution License (https://creativecommons.org/licenses/by/4.0/). See http://ivyspring.com/terms for full terms and conditions.

Received: 2020.10.02; Accepted: 2021.03.20; Published: 2021.04.03

\begin{abstract}
Implant-derived wear particles can be phagocytosed by local macrophages, triggering an inflammatory cascade that can drive the activation and recruitment of osteoclasts, thereby inducing peri-prosthetic osteolysis. Efforts to suppress pro-inflammatory cytokine release and osteoclastsogenesis thus represent primary approaches to treating and preventing such osteolysis. Sirtuin 3 (SIRT3) is a NAD+-dependent deacetylases that control diverse metabolic processes. However, whether SIRT3 could mitigate wear debris-induced osteolysis has not been reported. Herein we explored the impact of the SIRT3 on titanium particle-induced osteolysis. Tartrate resistant acid phosphatase (TRAP) staining revealed that the inhibition of SIRT3 suppressed nuclear factor-KB ligand (RANKL)-mediated osteoclasts activation in a dose-dependent fashion. Notably, inhibition of SIRT3 also suppressed matrix metallopeptidase 9 (MMP9) and nuclear factor of activated T-cell cytoplasmic 1 (NFATcl) expression at the mRNA and protein levels, while also inhibiting the mRNA expression of dendritic cell-specific transmembrane protein (DC-STAMP), ATPase $\mathrm{H}^{+}$Transporting V0 Subunit D2 (Atp6v0d2), TRAP and Cathepsin K (CTSK) . In addition, inhibition of SIRT3 suppressed titanium particle-induced tumor necrosis factor-alpha (TNF- $\alpha$ ), interleukin-1 $\beta$ (IL-1 $\beta$ ) and interleukin-6 (IL-6) expression and prevented titanium particle-induced osteolysis and bone loss in vivo. This inhibition of osteoclasts differentiation was found to be linked to the downregulation and reduced phosphorylation of JNK and ERK. Taken together, inhibition of SIRT3 may be a potential target for titanium particle-induced bone loss.
\end{abstract}

Key words: Sirtuin 3, peri-prosthetic osteolysis, osteoclasts, inflammatory factors

\section{Introduction}

Lower extremity total joint replacement surgery is associated with very positive outcomes, including a $>90 \%$ average $15-20$ year survival rate $[1,2]$. However, as rates of joint replacement surgery continue to rise, so too will rates of joint revision surgeries [3, 4]. A number of causes may contribute to the need for revision, with aseptic loosening accounting for 55\% and $31 \%$ of hip and knee revisions, respectively [5]. While the mechanistic basis for aseptic loosening remains to be clarified, wear particle-induced periprosthesis osteolysis is thought to be an important driver of this process [6]. Macrophages can take up wear particles, which subsequently stimulate them to produce a range of inflammatory factors including tumor necrosis factor- $\alpha$ (TNF- $\alpha$ ), interleukin-1 $\beta$ (IL-1 $\beta$ ), and interleukin-6 (IL-6), near the implant, thereby driving osteoclasts activation and pathological bone absorption [7]. Excessive differentiation of osteoclasts is also closely related to other serious diseases, such as rheumatoid arthritis and osteoporosis [8, 9]. Identifying novel molecular targets that can inhibit osteoclasts activation and differentiation may thus be 
an effective approach to treating of preventing bone loss-related diseases.

Osteoclasts are large multinuclear cells that form following macrophage/monocyte fusion and that facilitate bone resorption [10]. Osteoclasts precursor proliferation and differentiation can be driven by macrophage phage colony-stimulating factor (M-CSF) and nuclear factor- $\mathrm{kB}$ ligand (RANKL), ultimately inducing the proliferation, migration, and fusion of these cells and thereby inducing osteoclasts development $[11,12]$. RANKL is a transmembrane protein from the tumor necrosis factor (TNF) superfamily, which plays an important role in osteoclasts formation. When RANKL binds to its cognate receptor, RANK, the adaptor protein TNF receptor-associated factor 6 (TRAF6) is recruited [13, 14]. TRAF6, in turn, triggers a range of downstream MAPK and nuclear factor-kB (NF-kB) signaling pathways, ultimately resulting in nuclear NFATc1 activation, which is essential for osteoclasts differentiation and development [15-18]. A number of anti-osteoclastsic compounds have been described to date, including bisphosphonates [19], calcitonin [20], and denosumab [21]. These agents, however, exhibit significant side effects that hamper their clinical utility. For example, bisphosphonates can cause gastrointestinal symptoms, hypocalcemia, secondary hyperparathyroidism, and musculoskeletal pain [19]. It is thus important that novel approaches to regulating osteoclasts development and activation be developed that are both safe and efficacious.

Sirtuin family members are $\mathrm{NAD}^{+}$-dependent deacetylases that control diverse metabolic processes in cells and thereby function as essential regulators of survival, autophagy, growth, DNA repair and duplication, oxidative stress, and mitochondrial biogenesis [22-25]. There are seven known sirtuins (SIRT1-7), of which SIRT3 is known to be particularly important in the context of aging-related diseases such as cancer, cardiovascular disease, hearing loss, type 2 diabetes, and obesity [26]. Bone marrow metabolism and systemic metabolism are closely linked to one another $[27,28]$, but the role of SIRT3 in osteolysis is not clear, and further study of how SIRT3 regulates bone metabolism is thus essential. The present study was designed to assess the importance of SIRT3 as a regulator of osteolysis and to explore any mechanisms underlying its role in this process.

Herein, we demonstrated that the inhibition of SIRT3 suppressed the development of osteoclasts in response to RANKL stimulation and reduced the production of pro-inflammatory cytokines. Furthermore, SIRT3 inhibition reduced titanium particle-induced osteolysis in vivo, indicating that SIRT3 may serve as an important regulator of bone metabolism.

\section{Materials and Methods}

\section{Drugs and Reagents}

3-(1H-1,2,3-triazol-4-yl) pyridine (3-TYP) (purity $\geq 99 \%$ ) was obtained from Selleck (Shanghai, China) and dissolved in DMSO. PD184352 (CI-1040) was obtained from Selleck (Shanghai, China). Titanium (Ti) particles were purchased from Johnson Matthey Chemicals (MA, USA). Alpha modification of Eagle's medium (a-MEM), penicillin/streptomycin, and fetal bovine serum (FBS) were from HyClone (UT, USA). RANKL and M-CSF were from R\&D Systems (MN, USA). Primary antibodies specific for phospho-NF-kB p65 (\#3033), phospho-IkBa (\#2859), NF-kB p65 (\#8242), IkBa (\#4814), phospho-ERK (\#4370), phospho-p38 (\#4511), phospho-JNK (\#4668), ERK (\#4695), p38 (\#8690), and JNK (\#9252) were from Cell Signaling Technology (MA, USA). MMP9 (ab76003) and NFATc1 (ab25916), CTSK (ab178647), c-fos (ab208942) were from Abcam (Cambridge, UK). Antirabbit and anti-mouse HRP-conjugated secondary antibodies were from Multi Sciences (Shanghai, China).

\section{Cell culture and osteoclasts differentiation}

Primary bone marrow-derived macrophages (BMMs) were obtained using bone marrow isolated from the femurs and tibiae of 6-week-old female C57BL/ 6 mice. These cells were cultured in a-MEM containing $10 \% \mathrm{FBS}, 1 \%$ penicillin/streptomycin, and $30 \mathrm{ng} / \mathrm{mL}$ M-CSF in a $37^{\circ} \mathrm{C}, 5 \% \mathrm{CO} 2$ incubator until $90 \%$ confluent. Non-adherent cells were discarded after $24 \mathrm{~h}$, while the remaining adherent cells were cultured for an additional 3 days. BMMs were then plated at $8 \times 10^{4} /$ well in 24 -well plates (Corning, NY, USA), after which they were stimulated with RANKL $(50 \mathrm{ng} / \mathrm{mL}$ ) and M-CSF (30 ng/mL). Following a 6 day culture period, TRAP staining was conducted, and cells with $3+$ nuclei are considered mature osteoclasts.

\section{Cytotoxicity assay}

A CCK-8 assay kit (ApexBio, MA, USA) was used to assess the cytotoxic impact of 3-TYP on BMMs. Briefly, these cells were added to 96-well plates until adherent, at which time they were treated for $48 \mathrm{~h}$ with a range of 3-TYP concentrations. Next, 10 $\mu \mathrm{L}$ of CCK- 8 buffer was added per well for $2 \mathrm{~h}$ during which cells were incubated at $37^{\circ} \mathrm{C}$. Absorbance at $450 \mathrm{~nm}$ in each well was then assessed via microplate reader (BioTek, VT, USA).

\section{TRAP staining}

BMMs were plated at $8 \times 10^{4} /$ well in 24 -well 
plates, after which they were stimulated with RANKL $(50 \mathrm{ng} / \mathrm{mL})$ and M-CSF $(30 \mathrm{ng} / \mathrm{mL})$ in the presence of a range of 3-TYP concentrations $(0,25,50$ and $100 \mu \mathrm{M})$ to induce osteoclasts differentiation. The differentiation of these cells was then assessed via microscopy and TRAP staining conducted using a TRAP staining kit (Sigma-Aldrich, MO, USA). TRAPpositive cells with $3+$ nuclei were considered to be osteoclasts and these cells were counted using the Image J software (NIH, MD, USA).

\section{SIRT3 knockdown by siRNA}

To silence the SIRT3 gene, a siRNA targeting SIRT3 was designed and purchased from geneparma (Suzhou, China). Then special SIRT3 siRNA or non-binding control siRNA was transfected into BMMs using Lipofectamine RNAiMax reagent (Invitrogen, Carlsbad, CA, USA) according to the manufacturer's instructions. Briefly, BMMs were cultured in 6-well plates $\left(3 \times 10^{4} /\right.$ well $)$ in a-MEM medium before transfection, After $6 \mathrm{~h}$, the medium was replaced with a-MEM containing 10\% FBS. $48 \mathrm{~h}$ later, the efficiency of transfection was observed under fluorescence microscope. The sequence of SIRT3 siRNA oligonucleotide as follow: 5-GACCUUU GUAACAGCUACATT-3, the antisense is 5-UGUAG CUGUUACAAAGGUCTT -3.The sense of negative control siRNA is 5- UUCUCCGAACGUGUCACG UTT-3, the antisense is 5-ACGUGACACGUUCGGA GAATT-3.

\section{Podosome belt staining}

BMMs were plated at $8 \times 10^{4} /$ well in 24 -well plates, after which they were stimulated for 5 days with RANKL (50 $\mathrm{ng} / \mathrm{mL}$ ) and M-CSF $(30 \mathrm{ng} / \mathrm{mL})$ in the presence of a range of 3-TYP $(0,25,50$ and 100 $\mu \mathrm{M})$. Cells were then stained using DAPI and phalloidin, after which F-actin and cellular nuclei were imaged via fluorescence microscopy (Zeiss, Dresden, Germany).

\section{Resorption pit assay}

Resorption pit assays were conducted to assess osteoclasts functionality. Briefly, BMMs were plated at $8 \times 10^{4}$ /well in Osteo Assay Surface 24-well plates (Corning, USA)[29] , after which they were stimulated for 7 days with RANKL (50 ng/mL) and M-CSF (30 $\mathrm{ng} / \mathrm{mL})$ in the presence of a range of 3-TYP $(0,25,50$, $100 \mu \mathrm{M})$. After that, the cells were trypsinized and washed 3 times with PBS. The images were captured using an ordinary optical microscope (Zeiss), and Image J (NIH, MD, USA) was used to analyze the resorption area.

\section{RT-PCR}

BMMs were cultured at $1 \times 10^{5}$ cells/well in 6-well plates in a-MEM containing RANKL (50 $\mathrm{ng} / \mathrm{mL})$ and M-CSF $(30 \mathrm{ng} / \mathrm{mL})$. At appropriate time points, Trizol (Beyond, Shanghai, China) was used to extract total cellular RNA, and cDNA was then synthesized. Next, qRT-PCR reactions were conducted using a $10 \mu \mathrm{L}$ reaction volume containing 5 $\mu \mathrm{L}$ SYBR Green QPCR Master Mix (Yeasen, Shanghai, China), $3 \mu$ RNase-free $\mathrm{H}_{2} \mathrm{O}$ (Abcam, Cambridge, UK), $1 \mu \mathrm{lDNA}$, and $0.5 \mu \mathrm{L}$ each of the forward and reverse primers. Thermocycler settings were as follows: $95^{\circ} \mathrm{C}$ for 10 minutes; 40 cycles of $95^{\circ} \mathrm{C}$ for 10 $\mathrm{s}, 60^{\circ} \mathrm{C}$ for $20 \mathrm{~s}$, and $72{ }^{\circ} \mathrm{C}$ for $90 \mathrm{~s}$. GAPDH served as a normalization control, and primer sequences are shown in Table S1.

\section{Immunofluorescent staining}

Immunofluorescent staining for NFATc1 and MMP9. MMP9 and NFATc1 were detected via immunofluorescent staining. Briefly, cells were stained overnight at $4{ }^{\circ} \mathrm{C}$ with antibodies specific for MMP9 and NFATc1 (1:250; Abcam). Cells were then rinsed for 5 minutes with PBS, after which they were stained for $1 \mathrm{~h}$ with Goat Anti-Rabbit IgG H\&L (Alexa Fluor 647) at $37^{\circ} \mathrm{C}$. Phalloidin and DAPI were then used to counterstain the cytoskeleton and nuclei of these cells, and a fluorescence microscope (Zeiss) was used for cellular imaging.

\section{Western blot analysis}

BMMs were cultured at $6 \times 10^{5}$ cells/well in 6-well plates, and were cultured in the presence of RANKL (50 ng/mL), M-CSF (30 ng/mL), and a range of 3-TYP concentrations. Cells were then lysed with RIPA buffer, after which a BCA assay kit (Beyotime Biotechnology, Shanghai, China) was used to quantify protein levels in isolated samples. Protein was then separated via SDS-PAGE and transferred to nitrocellulose membranes (Beyotime) that were blocked for $1 \mathrm{~h}$ with QuickBlock ${ }^{\mathrm{TM}}$ blocking buffer (Beyotime). Blots were next probed overnight with primary antibodies at $4{ }^{\circ} \mathrm{C}$, and were washed thrice with TBST (10 minutes/wash) followed by a $1 \mathrm{~h}$ incubation with an appropriate secondary antibody. Protein bands were then detected via enhanced chemiluminescence (Sigma-Aldrich, MO, USA).

\section{Murine model of Ti particle-induced calvarial osteolysis}

The Institutional Animal Ethics Committee of the First Hospital of Soochow University approved all animal experiments described herein. Briefly, 40 female C57BL/6 mice (6-8 weeks old) were randomized into four treatment groups ( $\mathrm{n}=10 /$ group): (1) a sham group (PBS only), (2) a vehicle group, (3) a low-dose 3-TYP group (25 $\mathrm{mg} / \mathrm{kg}$ ), and (4) a high-dose 3-TYP group (50 mg/kg). 
All groups other than the sham group underwent a 20 mg Ti particle-stimulation procedure. Briefly, after anesthesia, make an $6 \mathrm{~mm}$ sagittal incision in the middle of the skull, $20 \mathrm{mg}$ Ti particles were evenly embedded under the periosteum on the surface of the parietal bone, and then the incision was sutured. Whereas in the sham group, the incision was closed immediately with no further intervention. All mice were intraperitoneally injected with the appropriate solutions every other day for 2 weeks, after which animals were euthanized and skull caps were collected and fixed using $4 \%$ paraformaldehyde.

\section{Micro-CT imaging}

Murine skull samples were first fixed for $24 \mathrm{~h}$ in $10 \%$ neutral formalin, after which a Skyscan 1176 micro-CT (Aartselaar, Belgium) was used to conduct micro-computed tomography (micro-CT) scans of these samples ( $\mathrm{n}=5$ /group) with the following settings: $9 \mu \mathrm{m}$ equidistant resolution, $50 \mathrm{kV}$, and 200 $\mu \mathrm{A}$ energy. Three-dimensional reconstructions were conducted using X-ray CT analyzer (SkyScan), which was also used for optical measurements of assorted tissue morphology parameters including the bone volume/tissue volume (BV/TV), bone mineral density (BMD), number of pores, and percentage of porosity.

\section{Histological analysis}

After collection, samples were decalcified for 4 weeks in a $10 \%$ EDTA solution at $37^{\circ} \mathrm{C}$, after which they were paraffin-embedded, and sections were prepared with an appropriate instrument (Leica 2135, Germany). These sections were subjected to TRAP and hematoxylin and eosin (H\&E) staining, after which an axiovert 40C optical microscope (Zeiss, Germany) was used for sample analysis.

\section{Statistical analysis}

Data are given as means \pm standard deviation (SD), and were analyzed using GraphPad Prism 8.0 (GraphPad Software Inc. CA, USA). Data were compared between groups via ANOVAs with TukeyKramer's post-hoc test. ${ }^{*} \mathrm{p}<0.05,{ }^{* *} \mathrm{p}<0.01,{ }^{* * *} \mathrm{p}<$ 0.005 .

\section{Results}

\section{Effect of SIRT3 on the development of RANKL-induced osteoclasts in vitro}

We firstly found that SIRT3 expression was increased significantly in RANKL-induced osteoclasts relative to levels in untreated BMMs (Fig. 1A-B). SIRT3 expression rose with osteoclasts activation, indicating that SIRT3 may play an important regulatory role in this process. We additionally began by assessing the ability of the selective SIRT3 inhibitor 3-TYP (Fig. 1C) to induce direct cytotoxicity in murine BMMs over a $48 \mathrm{~h}$ culture period in vitro, revealing that doses under $100 \mu \mathrm{M}$ had no impact on cell viability (Fig. 2D-E). We also demonstrated that 3-TYP suppressed SIRT3 expression during RANKLinduced osteoclasts formation (Fig. S1). We then explored the ability of 3-TYP to influence osteoclasts differentiation by treating BMMs with RANKL (50 $\mathrm{ng} / \mathrm{mL}$ ), M-CSF (30 ng/mL), and a range of 3-TYP concentrations, revealing that 3-TYP inhibits osteoclasts formation in a concentration-dependent manner (Fig. 1F-G). To more fully understand how 3-TYP inhibits osteoclastsogenesis, BMMs were then treated with $100 \mu \mathrm{M} 3$-TYP for different time intervals during the differentiation process $(0-2,2-4,4-6$, and 0 6 days). While 3-TYP treatment from days 0-2 dramatically inhibited osteoclasts differentiation, treatment from days 4-6 did not affect this differentiation process, suggesting that 3-TYP functions to suppress the early stages of RANKL-induced osteoclasts differentiation without causing cytotoxicity (Fig. S2). Finally, we further demonstrate the SIRT3-dependent function involved in the formation of osteoclasts, SIRT3 siRNA was employed to knock down its endogenous expression and determine the effects on RANKL-induced osteoclastogenesis. Western blotting confirmed that SIRT3 siRNA could significantly reduce the endogenous expression of SIRT3 (Fig. 1H-I). In addition, SIRT3 siRNA reduces the number of osteoclasts, compared with treatment with control siRNA (Fig. 1J-K). Collectively, these results indicate that inhibition of SIRT3 inhibits RANKL-induced activation of osteoclasts.

\section{Inhibition of SIRT3 suppresses F-actin ring formation and bone resorption}

We next utilized a pit formation assay as a means of gauging the impact of SIRT3 on bone resorption, revealing that 3-TYP reduced bone resorption area in a dose-dependent fashion (Fig. 2A-B). Marked reductions in bone resorption area were observed following sample pretreatment with 3-TYP, particularly at high doses $(100 \mu \mathrm{M})$. At the same time, TRAP staining was used to record osteoclasts (Fig. 2C-D). Phalloidin was also used to stain these cells in order to assess F-actin ring formation as a metric for osteoclasts function [30,31]. This analysis indicated that 3-TYP pretreatment was sufficient to reduce F-actin ring formation (Fig. 2E-F), consistent with the bone resorption assay results. 
A

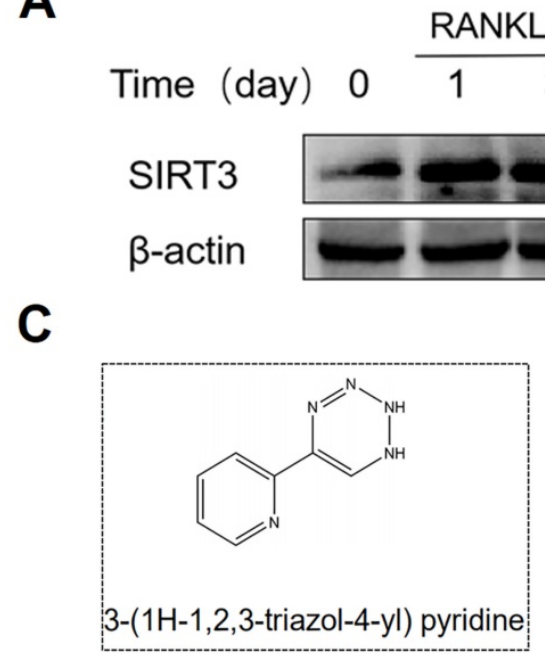

$\mathbf{F}$
D
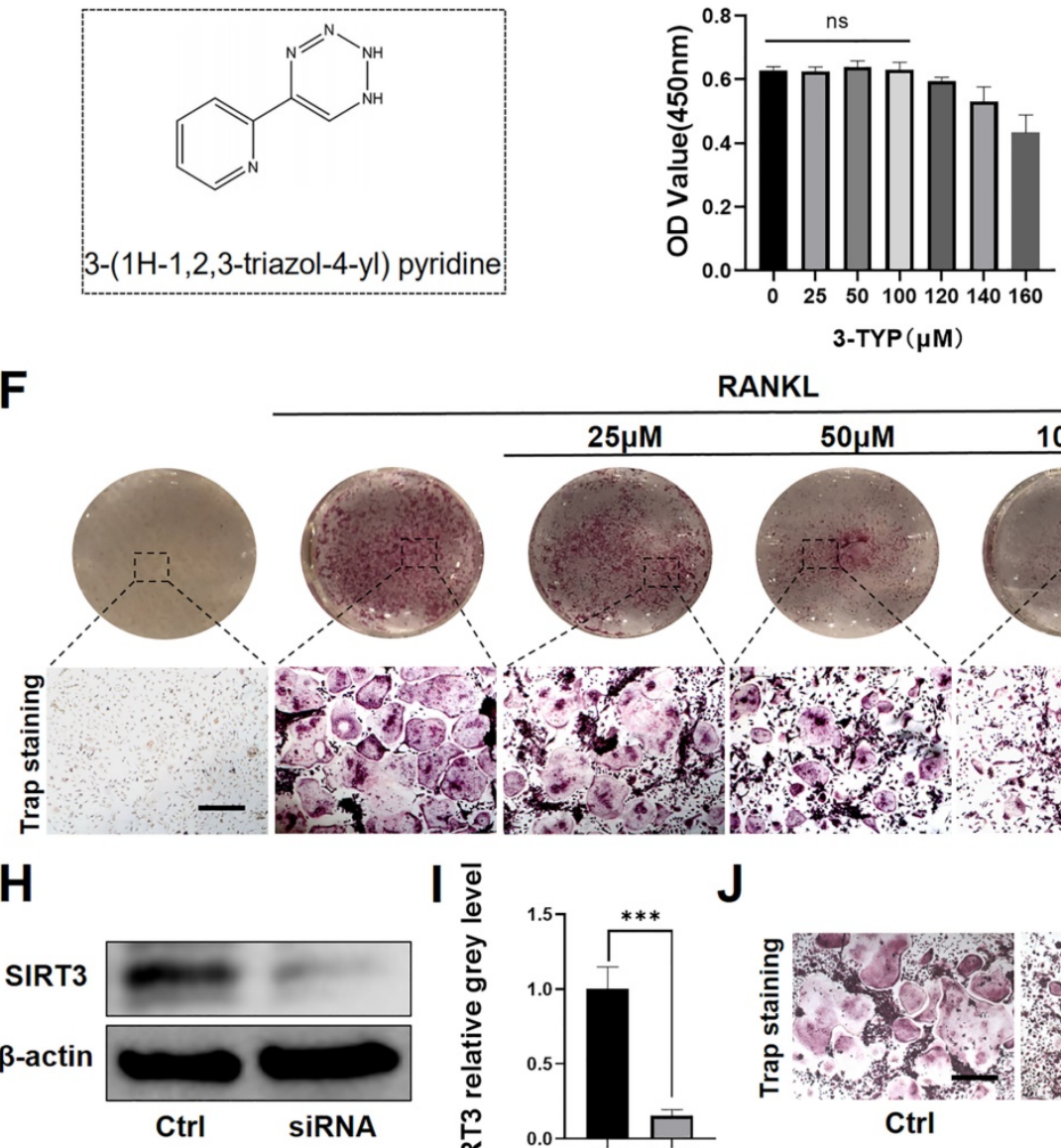

RANKL
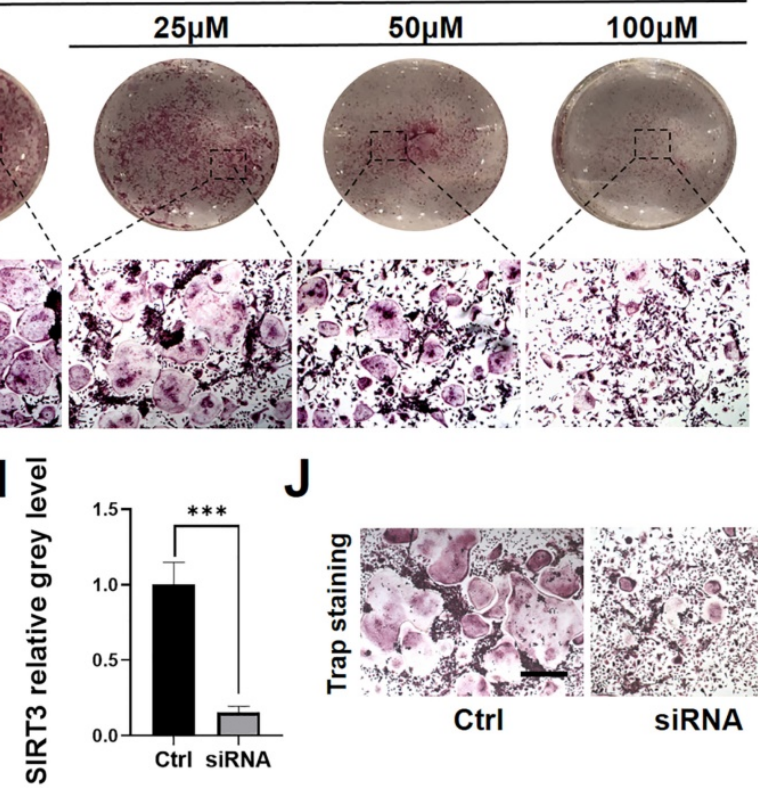

CCK-8

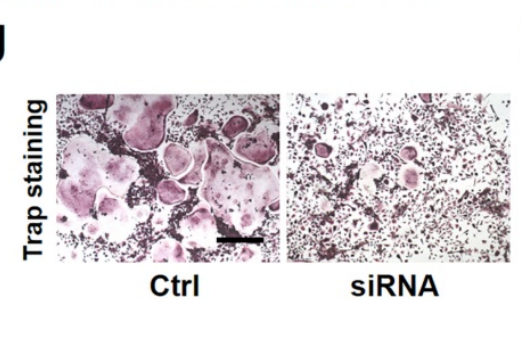

G
B
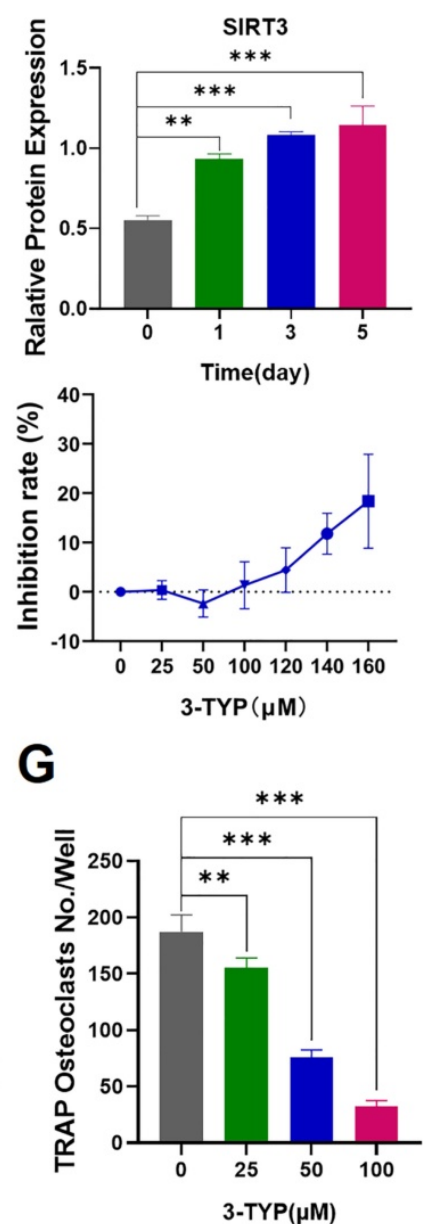

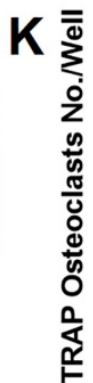

Figures 1. Effect of SIRT3 on the development of RANKL-induced osteoclasts in vitro. (A) SIRT3 expression was assessed via Western blotting at the indicated time points. (B) Results of western blotting are quantified. (C) 3-TYP structure and chemical formula. (D) Bone marrow macrophages (BMMs) were cultured in $\alpha-M E M$ containing RANKL, M-CSF and treated with different concentrations of 3-TYP, CCK-8 kit was used to detect cell proliferation. (E) Inhibition rate of 3-TYP on BMMs. (F) TRAP staining was used to analyze osteoclasts differentiation, revealing that 3-TYP inhibits osteoclastogenesis in a dose-dependent fashion. (G) TRAP-positive multinucleated cells and osteoclasts (nuclei $\geq 3$ ) in each well were quantified. (F) BMMs were transfected with SIRT3 siRNA or scrambled siRNA (control). (I) Results of western blotting are quantified. (J) TRAP staining. (K) TRAP-positive multinucleated cells were quantified. Scale bar: $100 \mu \mathrm{m}$. Data are means $\pm \operatorname{SD}\left({ }^{*} \mathrm{p}<0.05,{ }^{* *} \mathrm{p}<0.01,{ }^{* * * *} \mathrm{p}<0.005, \mathrm{n}=3\right)$.

\section{Inhibition of SIRT3 suppresses osteoclast- associated gene and/or protein expression}

To further assess the impact of SIRT3 on osteoclastsogenesis, we next analyzed the expression of key osteoclast-associated genes such as TRAP, MMP9, DC-STAMP, Atp6v0d2, CTSK and NFATc1, we found that 3-TYP suppressed the expression of these genes in a dose-dependent fashion (Fig. 3A-F). Immunofluorescent staining of the actin rings and NFATc1 or MMP9 shows that inhibition of SIRT3 reduces the size of osteoclasts and expression of
NFATc1 and MMP-9 (Fig. 3G-H). Western blotting confirmed that the expressions of osteoclastic makers such as MMP-9, c-fos and CTSK are down regulated after exposure to 3-TYP and SIRT3 siRNA (Fig. 3I-J).

\section{Inhibition of SIRT3 prevents RANKL-induced MAPK signaling}

To assess the mechanistic basis whereby inhibition of SIRT3 suppresses osteoclastic differentiation, we next analyzed the NF-KB, MAPK, and PI3K/AKT pathways in these cells via Western blotting given that these pathways are essential 


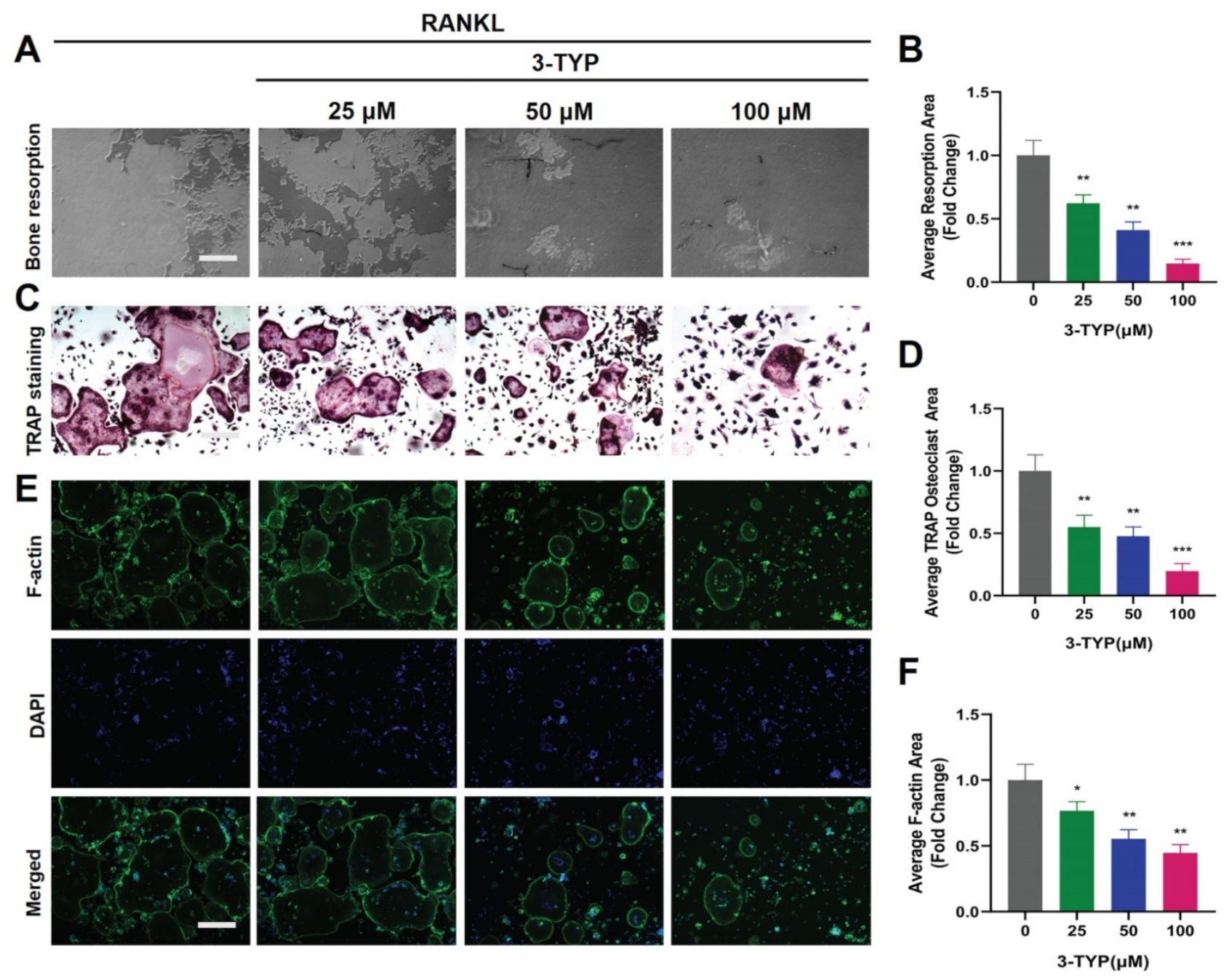

Figures 2. Inhibition of SIRT3 suppresses F-actin ring formation and bone resorption. (A) Resorption pits were assessed via inverted microscope. (B) Image J was used to measure pit area values. (C) TRAP staining was used to analyze osteoclasts differentiation (D) TRAP positive cells are quantified. (E) Osteoclasts were stained for F-actin (green) and nuclei (blue). (F) Quantification of the F-actin ring number per well. Scale bar: $100 \mu \mathrm{m}$. Data are means $\pm S D\left(* \mathrm{p}<0.05, * * \mathrm{p}<0.01, * * *{ }^{*}<0.005, \mathrm{n}=3\right)$.

regulators of this differentiation process. With respect to MAPK signaling, 3-TYP pretreatment markedly inhibited both ERK and JNK phosphorylation without altering total protein levels (Fig. 4A). In contrast, 3-TYP did not significantly alter the phosphorylation of P65, IKBa, PI3K/ AKT in these cells, suggesting that NF-KB and PI3K/AKT signaling were unaffected by this inhibitor (Fig. 4B). Figures 4C-H are the quantitative results of pathway-related proteins respectively. To further demonstrate that these changes in JNK and ERK signaling can account for the observed changes in osteoclastogenesis. We screened the ERK inhibitor PD184352 (CI-1040) to support the important role of ERK in osteoclasts activation. At 15 minutes, we found that CI-1040 inhibits the phosphorylation of ERK (Fig. 4I). Simultaneously, TRAP staining showed that CI-1040 significantly reduced TRAP-positive cells compared with the control, and the combination of CI-1040 and 3-TYP inhibited osteoclasts more obviously, which was attributed to the important role of the ERK signaling pathway in osteoclasts activation (Fig. 4J). Together, these data suggested that inhibition of SIRT3 suppresses RANKL-induced osteoclastsogenesis via disrupting MAPK signaling.

\section{Inhibition of SIRT3 dampens Ti particle- induced inflammatory cytokine production}

Pro-inflammatory cytokines are important mediators of osteoclasts development and survival $[32,33]$, and macrophages are the primary producers of wear debris-induced pro-inflammatory cytokine production in the context of osteolysis. Above all, we evaluated the cytotoxicity of different concentrations of titanium particles on RAW264.7 macrophages, and revealed that doses below $0.1 \mathrm{mg} / \mathrm{L}$ have no effect on cell viability (Fig. 5A). The inhibition rate is shown in the Figure 5B. As such, we next stimulated RAW264.7 macrophages with $\mathrm{Ti}$ particles $(0.1 \mathrm{mg} / \mathrm{mL})$ and a range of 3-TYP concentrations and explored 
consequent cytokine production. We found that $\mathrm{Ti}$ particles induced significant increases in levels of inflammatory factors including IL-6, TNF- $\alpha$ and IL-1 $\beta$ (Fig. 5C-E), whereas 3-TYP treatment inhibited the production of all of these cytokines at the RNA and protein levels (Fig. 5F). Protein quantification is shown in Figure 5G-I. This suggested that 3-TYP functions as an inhibitor of Ti-induced inflammatory activity.

\section{Inhibition of SIRT3 prevents Ti particle- induced osteolysis and bone loss in vivo}

Given the promising efficacy of inhibition of SIRT3 as an inhibitor of osteoclastsogenesis and inflammation in vitro, we next assessed its efficacy in vivo by establishing a $\mathrm{Ti}$ particle-induced murine calvaria osteolysis model [34], which is commonly utilized to analyze aseptic loosening. Particle-induced erosion resulted in the formation of large lacunae (Fig. 6A), whereas 3-TYP treatment markedly alleviated this destruction. Indeed, bone parameter analyses revealed that BMD was significantly decreased in the vehicle group relative to the sham control group, whereas 3-TYP treatment prevented this reduction, particularly in animals administered a high 3-TYP dose (Fig. 6B). Mice that had been treated with 3-TYP also exhibited an increased bone volume/tissue volume $(\mathrm{BV} / \mathrm{TV})$ relative to the vehicle group (Fig. $6 \mathrm{C})$, and exhibited fewer percentage of porosity and reduced number of porosity (Fig. 6D-E).
A

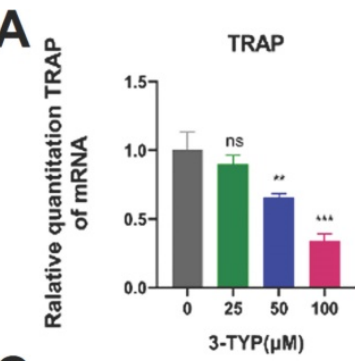

C

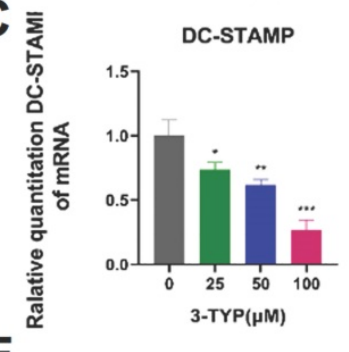

E

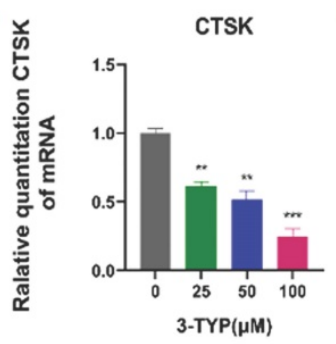

B

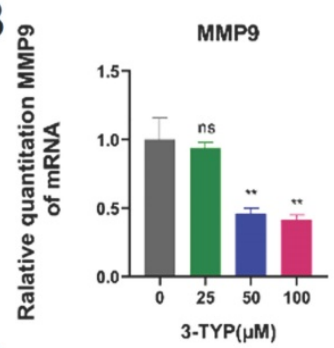

D

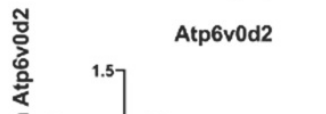

$\mathbf{F}$

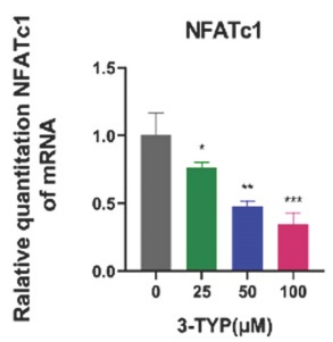

$\mathbf{G}$
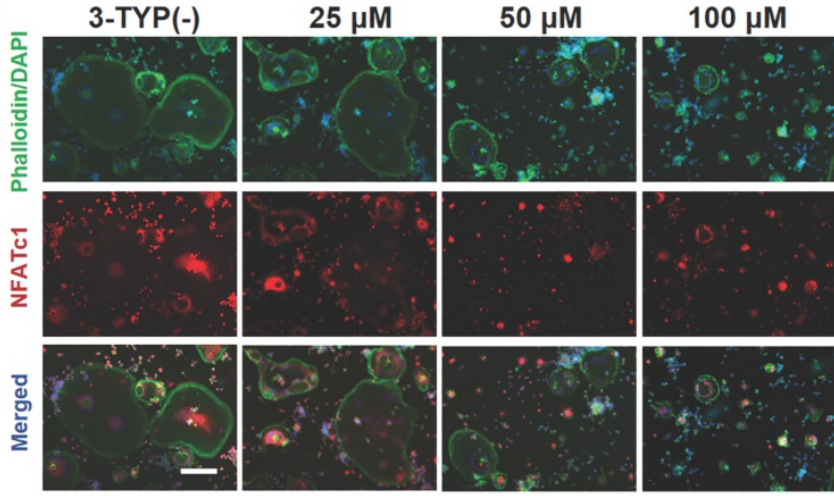

$\mathbf{H}$
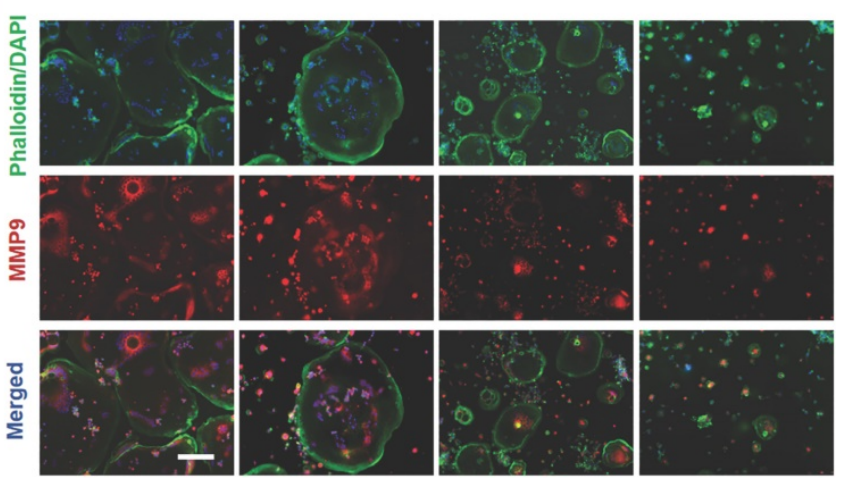

$\mathbf{J}$

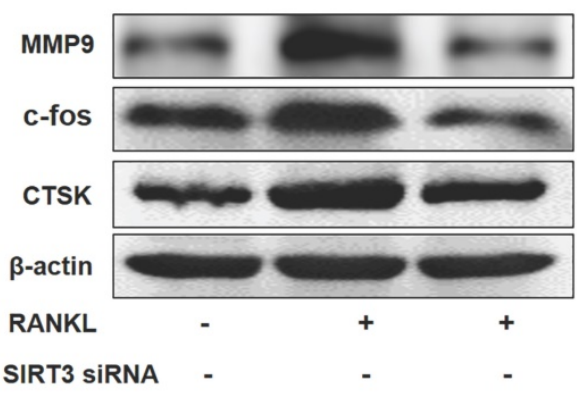

Figures 3. Inhibition of SIRT3 suppresses RANKL-induced osteoclast- associated protein and/or gene expression. (A-F) Expression osteoclast-associated genes (TRAP, MMP9, DC-STAMP, Atp6v0d2, CTSK and NFATc1) in BMMs following treatment for $24 \mathrm{~h}$ with a range of 3-TYP concentrations. (G-H) NFATcl and MMP9 expression was assessed via immunofluorescent analyses. (I) MMP9, c-fos and CTSK expression was assessed via Western blotting under 3-TYP treatment. (J) MMP9, c-fos and CTSK protein expression under SIRT3 siRNA treatment. Scale bars: $50 \mu \mathrm{m}$. Data are means \pm SD $(* \mathrm{p}<0.05, * * \mathrm{p}<0.01, * * * \mathrm{p}<0.005, \mathrm{n}=3)$. 


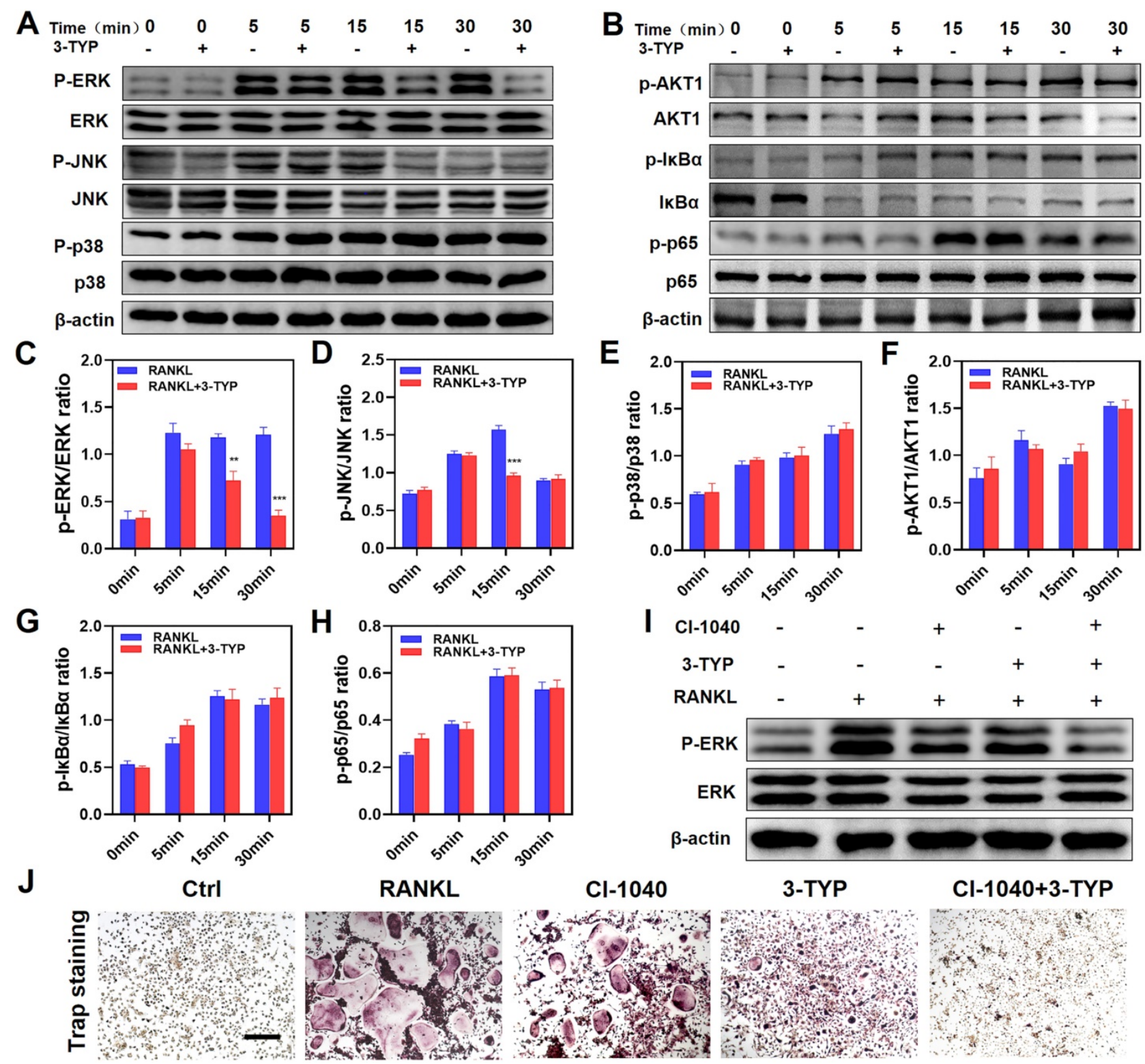

Figures 4. Inhibition of SIRT3 inhibits RANKL-induced MAPK signaling. (A) BMMs were treated for $12 \mathrm{~h}$ with 3-TYP, after which they were treated for 0-30 minutes with RANKL $(50 \mathrm{ng} / \mathrm{mL})$ and M-CSF $(30 \mathrm{ng} / \mathrm{mL})$. Western blotting was then used to measure ERK, JNK and p38 expression and phosphorylation. (C-E) Western blotting data were quantified respectively. (B) AKT1, IKBa and $\mathrm{p} 65$ expression and activation were assessed via Western blotting. (F-H) Western blotting data were quantified respectively. (I) ERK expression and phosphorylation at 15 minutes with RANKL $(50 \mathrm{ng} / \mathrm{mL})$ and M-CSF $(30 \mathrm{ng} / \mathrm{mL})$. (J) TRAP staining was used to analyze osteoclasts differentiation, revealing that ERK signaling play a significant role in osteoclastogenesis. Scale bars: $100 \mu \mathrm{m}$. Data are means \pm SD $\left(*_{p}<0.05, * * p<0.01, * * * p<0.005, n=3\right)$.

Further histological analyses confirmed that inhibition of SIRT3 was able to prevent Ti particle-induced osteolysis (Fig. 7A). Analyses of H\&E-stained sections indicated that the erosion surface area had increased by nine-fold in the vehicle group relative to the sham-operated control group, and fibrous tissue thickness had increased by seven-fold in vehicle group samples. Importantly, 3-TYP treatment dramatically reduced the erosion surface and fibrous tissue area relative to that observed in vehicle group animals (Fig. 7C-D). TRAP staining results revealed that relative to vehicle group animals, numbers of TRAP-positive cells in the 3-TYP treatment group were significantly reduced (Fig. 7E), as was percentage of osteoclasts per bone surface (OCs/BS, \%) (Fig. 7F).

\section{Discussion}

Peri-prosthetic osteolysis and associated aseptic loosening are the most common long-term complications of joint replacement surgery, increasing the complexity of arthroplasty procedures [35]. While the mechanistic basis for such osteolysis remains incompletely understood, osteoclast-mediated bone resorption is known to be a primary driver of this process. Inhibiting osteoclasts activation and associated inflammation thus represent viable approaches to preventing or treating wear debris- 
induced peri-prosthetic osteolysis. In the present study, we determined that activated osteoclasts upregulate SIRT3, suggesting it may be involved in their differentiation and/or activation. We therefore treated osteoclasts with the SIRT3-selective 3-TYP inhibitor. Concentrations of 3-TYP that were under $100 \mu \mathrm{M}$ did not adversely affect BMMs proliferation or survival, whereas in bone resorption assays we found that 3-TYP suppressed osteoclasts function in a dose-dependent fashion. Importantly, H\&E staining and micro-CT analyses showed that 3-TYP was sufficient to prevent bone loss in vivo in a $\mathrm{Ti}$ particle-induced osteolysis model system. We also found that 3-TYP treatment reduced inflammatory cytokine production, which is noteworthy owing to the pathogenic role of these molecules in the context of osteolysis. In summary, these results indicated that inhibition of SIRT3 plays a key role in suppressing osteoclasts activation and inflammatory cytokine production, thereby suppressing Ti particle-induced bone loss.
We additionally assessed the mechanisms whereby 3-TYP inhibits osteoclasts differentiation and activation. The MAPK, NF-KB and PI3K/AKT signaling pathways are the primary pathways that govern osteoclastsogenesis and subsequent osteoclasts maturation, and these pathways are also closely linked to pro-inflammatory cytokine signaling [35-38]. The signaling activity of MAPK proteins including ERK, JNK, and p38 is essential to the differentiation and activation of normal osteoclasts [37]. At a functional level, ERK activation can enhance the expression of the collagenase MMP-9, which can in turn facilitate osteoclasts migration and bone resorption [39]. RANKL-induced activation of JNK leads to the phosphorylation of c-Jun, which complexes with c-Fos to yield an active transcription factor that is essential for osteoclastsic differentiation [40]. Herein, we found that inhibition of SIRT3 was sufficient to inhibit RANKL-induced MAPK signaling via suppressing ERK and JNK activation, whereas did not affect NF-KB and PI3K/AKT pathway signaling.
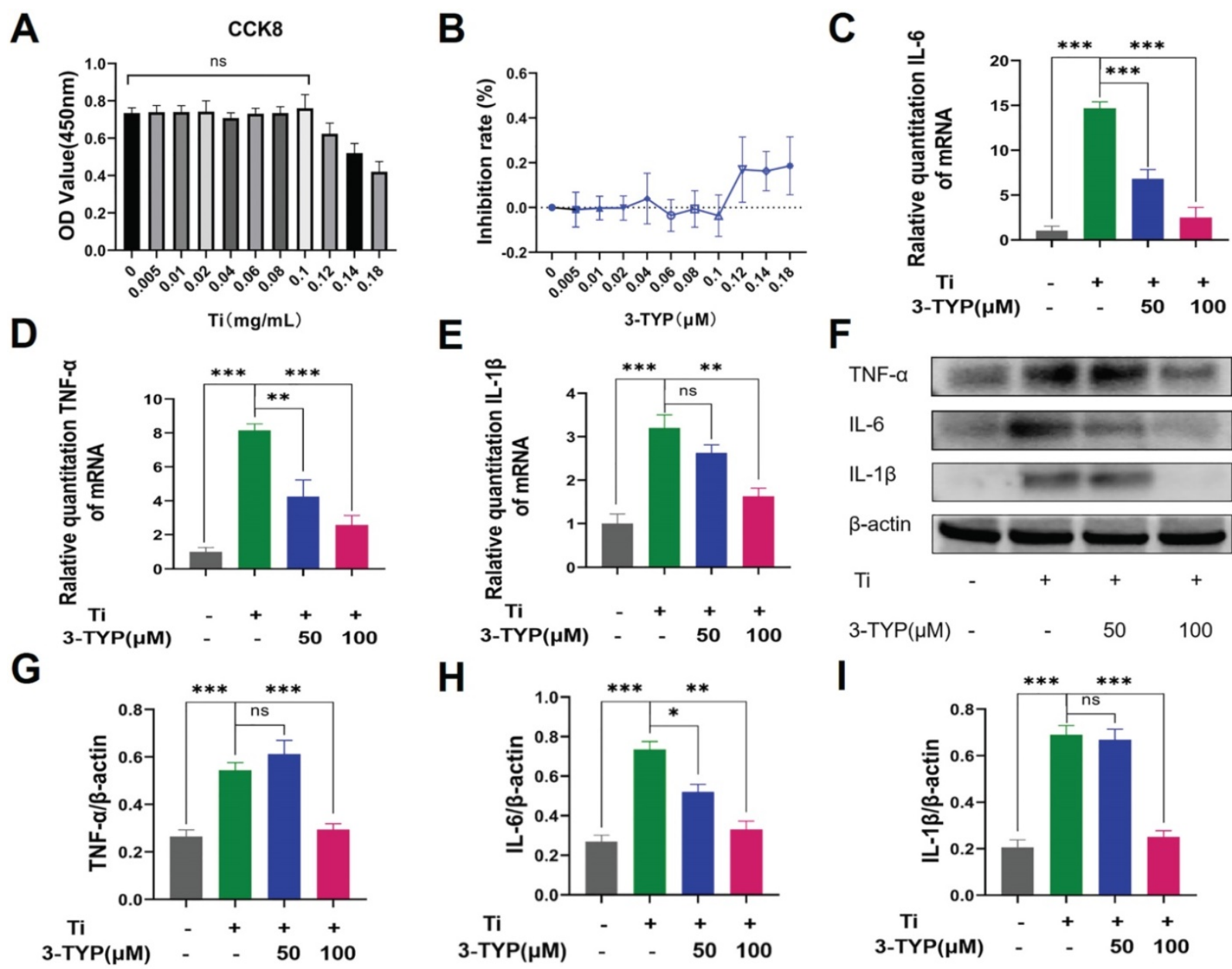

E
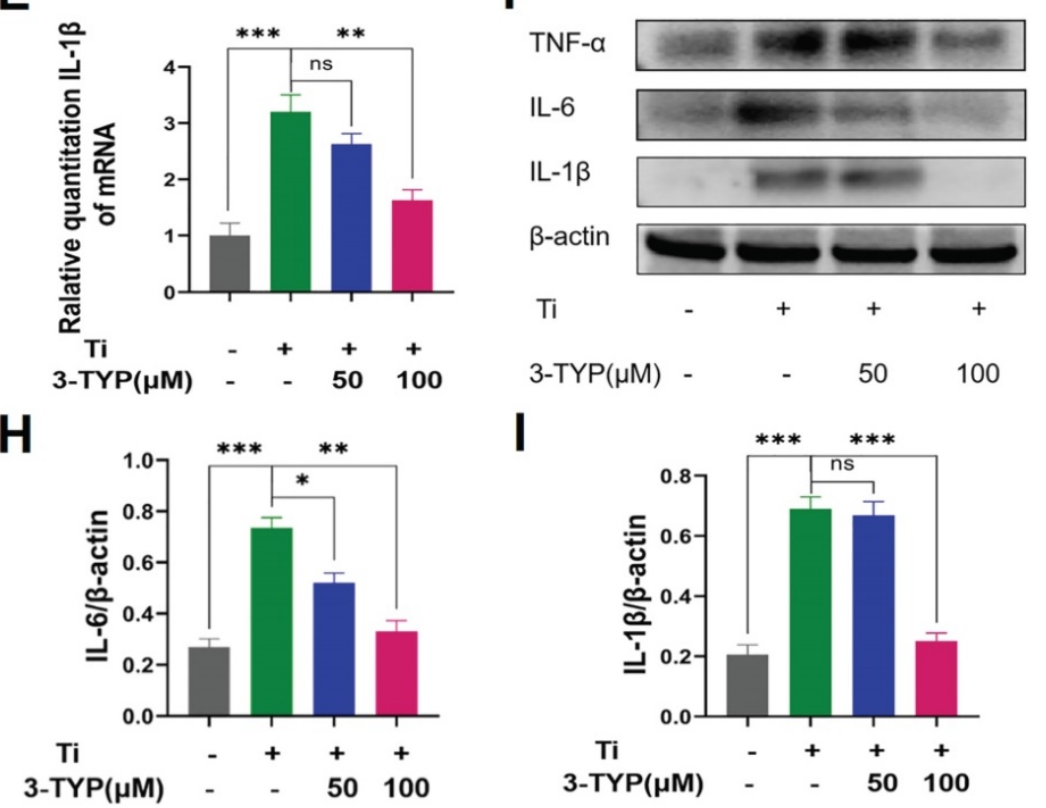

Figures 5. Inhibition of SIRT3 suppresses inflammatory cytokine release. (A) RAW 264.7 macrophages were cultured in different concentrations of the Ti particles for 72 hours. Cell viability was tested using cck-8. (B) Inhibition rate of Ti particles on RAW 264.7 macrophages. (C-E) RAW 264.7 macrophages were treated for 12 h with a range of 3-TYP doses, after which they were combined with Ti particles $(0.1 \mathrm{mg} / \mathrm{mL})$. The expression of pro-inflammatory cytokine genes (IL-6, TNF- $\alpha$ and IL- $1 \beta)$ was then assessed via RT-PCR. (F) Following a $24 \mathrm{~h}$ pretreatment with the indicated 3-TYP concentrations, RAW 264.7 macrophages were treated with Ti particles (0.1 mg/mL), and inflammatory cytokine levels (TNF- $\alpha$, IL- 6 and L-1 $\beta$ ) were assessed via Western blotting. (G - I) Western blotting data were quantified. Data are means $\pm S D(* p<0.05$, $* * p<0.01$, $* * * p<0.005$, $\mathrm{n}=3)$. 
A

Ti-particles
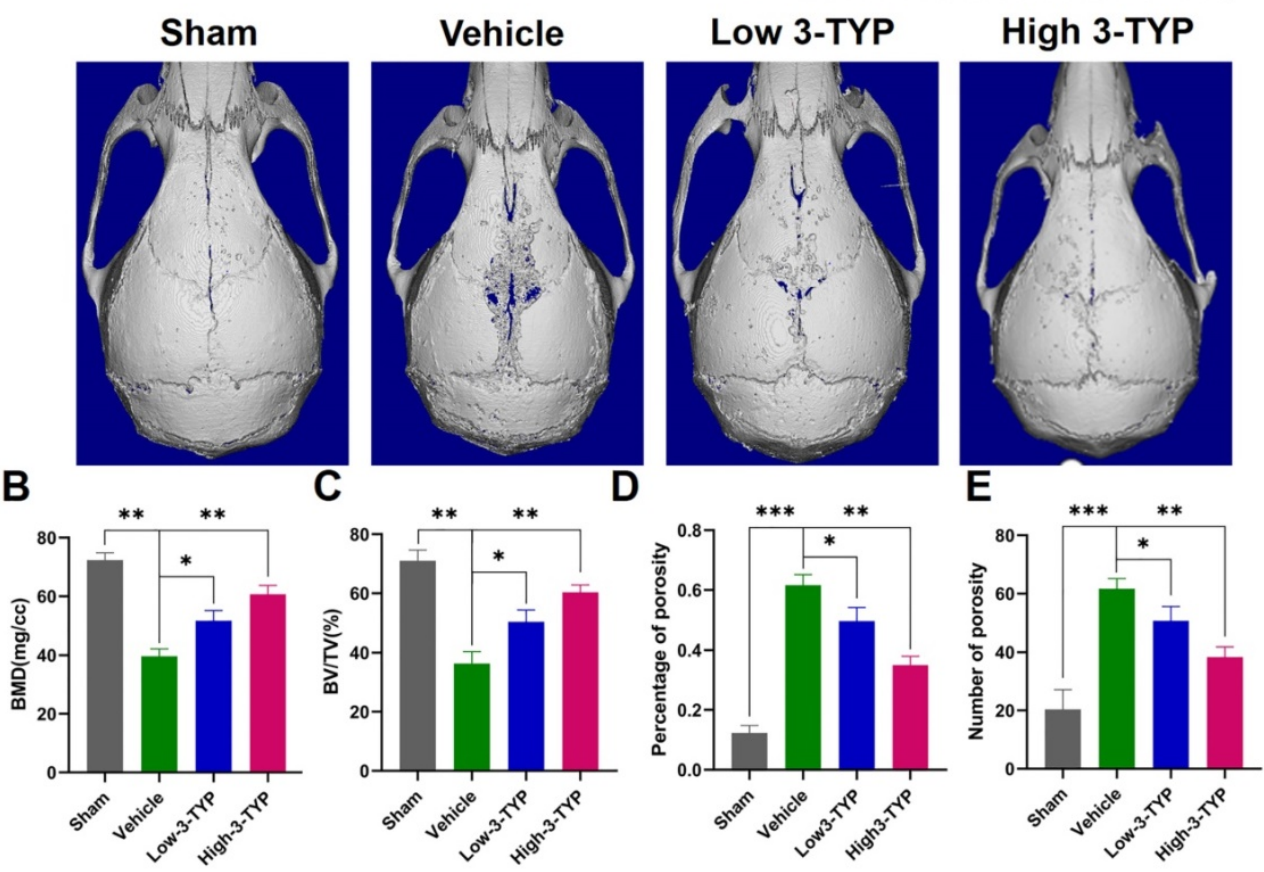

Figures 6. Inhibition of SIRT3 prevents Ti particle-induced osteolysis and bone loss in vivo. (A) Micro-CT analyses of calvaria samples from the indicated treatment groups. (B) Bone mineral density (BMD) values, (C) bone volume/tissue volume (BV/TV) values, (D) percentage of porosity and (E) number of porosity were quantified to assess bone microstructure. Data are means \pm SD $(* p<0.05, * * p<0.01, * * * p<0.005, n=5)$.
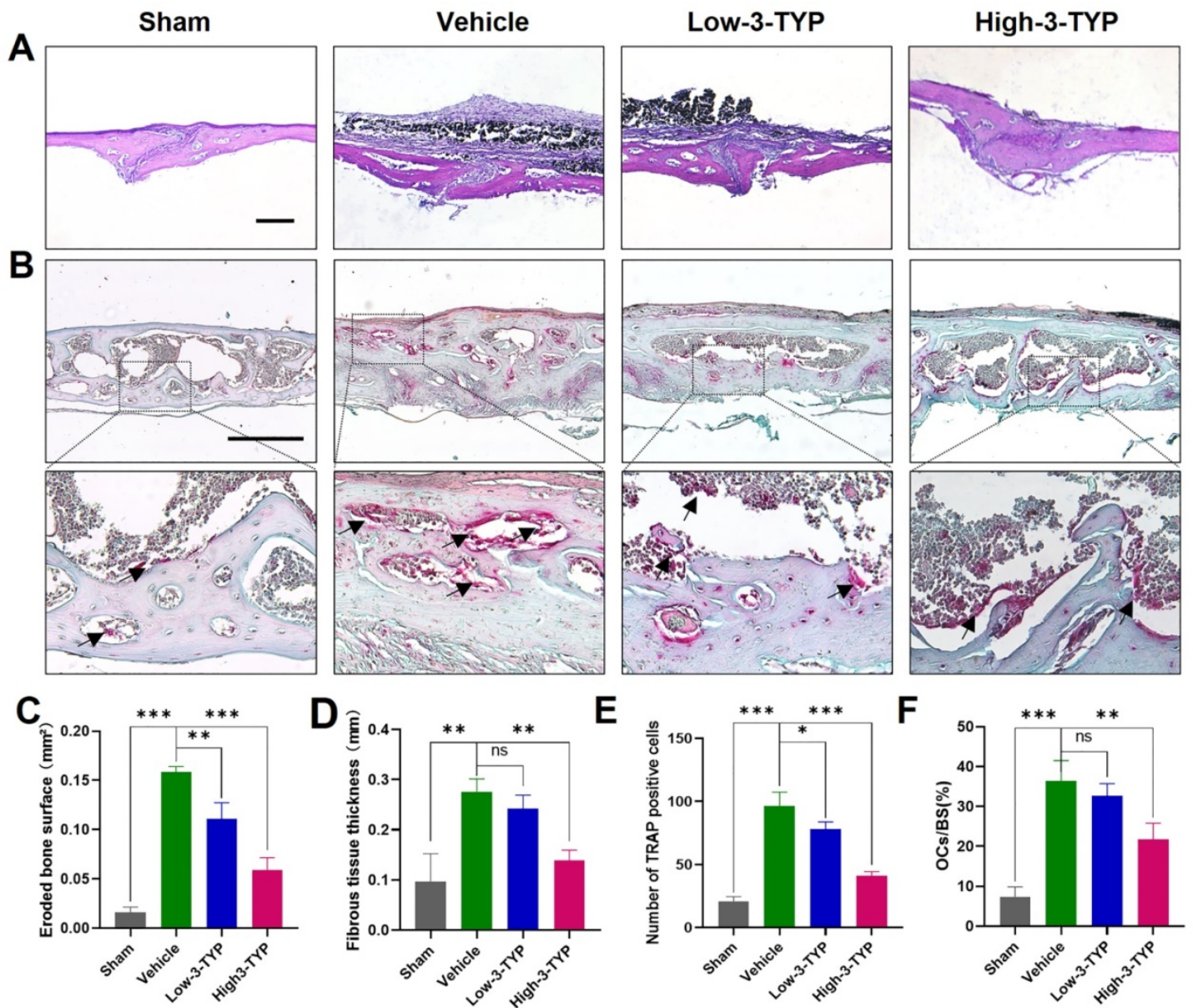

Figures 7. Inhibition of SIRT3 inhibits Ti particle-induced bone loss and reduced osteoclasts number. (A-B) H\&E and TRAP staining were conducted. (C) Eroded bone surface, (D) Fibrous tissue thickness, $(E)$ numbers of TRAP-positive cells, and (F) percentage of osteoclasts per bone surface (OCs/BS, \%) were determined. Scale bar: 200 $\mu \mathrm{m}$. Data are means \pm SD $(* \mathrm{p}<0.05, * * \mathrm{p}<0.01, * * * \mathrm{p}<0.005, \mathrm{n}=5)$. 


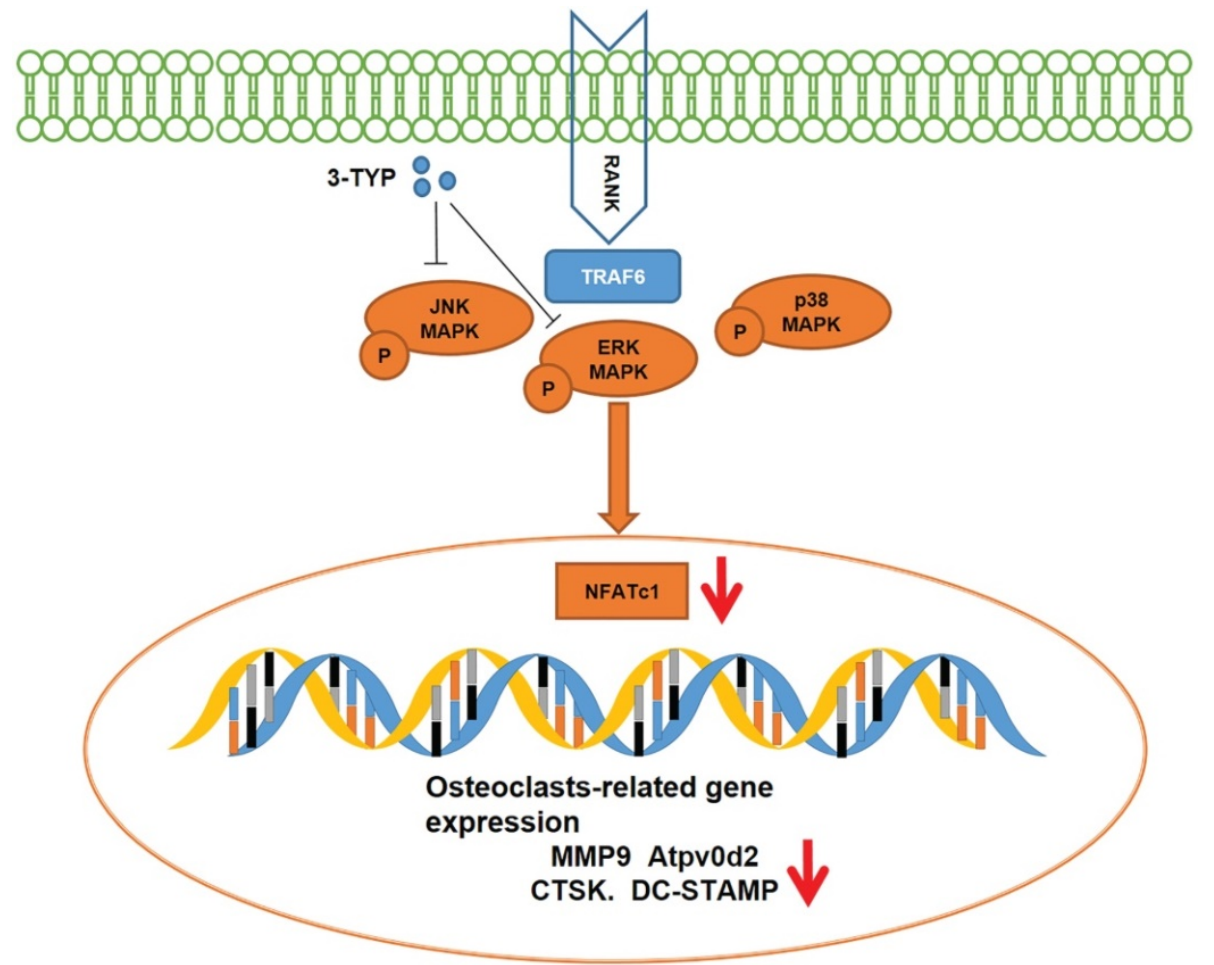

Figures 8. Schematic model of the signaling mechanism of 3-TYP inhibitory effect on Ti particle-induced skull osteolysis.

SIRT3 functions as a deacetylase responsible for the majority of mitochondrial lysine acetylation [41, 42], and it has been linked to functional roles in a range of pathogenic contexts such as cancer, obesity, type 2-diabetes, cardiovascular disease, and hearing loss. The role of SIRT3 in bone metabolism, however, has remained controversial. Kim et al. [43] who believed SIRT3 activates mitochondrial SOD activation through deacetylation, reduces ROS production, thereby reducing bone breakdown caused by osteoclasts activation. Huh et al. [44] found that SIRT3-/- mice had increased osteoclasts. However, Ho et al. [45] in contrast found that SIRT3 was a positive regulator of adipogenesis and osteoclastogenesis and a negative regulator of skeletal homeostasis. Our research is consistent with Ho et al. who believe that SIRT3 plays a positive role in osteoclasts activation. Moreover, Inhibition of SIRT3 prevents the pathological bone destruction by reducing the excessive activation of osteoclasts in the $\mathrm{Ti}$ particle-induced mouse osteolysis model. Currently, we are not sure what caused the difference, it may be related to the process of Ti particle-induced osteolysis, which involves macrophages engulfing titanium particles and inducing inflammatory cytokine mainly include TNF- $\alpha$, IL-1 $\beta$ and IL-6, these inflammatory factors play an important role in driving osteoclasts activation and pathological bone resorption [33].

Bone homeostasis is maintained by a balance between osteoclasts and osteoblasts activation in vivo, and disruptions in this homeostasis can result in serious metabolic bone disorders [46-48]. Osteolysis can thus be effectively treated by mediating bone formation. Wear particles have been shown to impair the activation and viability of osteoblasts [49]. Our data, however, do not rule out the possibility that inhibition of SIRT3 may impact osteoblastogenesis in addition to impacting osteoclasts differentiation. Indeed, Ding et al. [50] found SIRT3 to control mitochondrial function and to thereby serve as a key regulator of osteoblastic differentiation, indicating that overexpressing SIRT3 may thus drive bone formation via inducing osteoblasts development. As such, ongoing work in our laboratory seeks to resolve the impact of SIRT3 on osteoblasts differentiation in this experimental context.

There are multiple limitations to the present study. For one, our animal model is inconsistent with true peri-prosthetic osteolysis in human patients, as in humans this process occurs over extended periods of time due to the gradual accumulation of wear particles, unlike in our experimental model. Secondly, we only assessed local histological alterations within the skull and did not assess these animals for potential systemic side effects. Peri-prosthetic osteolysis is a complex process that requires cross-talk and cooperation among multiple cell types including osteoclasts and osteoblasts. Future research regarding the role of SIRT3 in osteoblasts is thus warranted. 
In summary, our results confirmed that the inhibition of SIRT3 can decrease Ti particle-induced bone resorption, suppress osteoclastsic differentiation, and decrease inflammatory cytokine production. SIRT3 may therefore represent a viable therapeutic target in the context of peri-prosthetic osteolysis or other diseases associated with inflammation and osteoclastsic differentiation.

\section{Supplementary Material}

Supplementary figures and tables. http://www.ijbs.com/v17p1382s1.pdf

\section{Acknowledgements}

This work is supported by grants from the National Natural Science Foundation of China (No. $82072425,82072498,81873991,81873990,81672238$ and 81472077), the Young Medical Talents of Jiangsu Province (No. QNRC2016751), the Natural Science Foundation of Jiangsu Province (No. BK20180001), the Priority Academic Program Development of Jiangsu Higher Education Institutions (PAPD), the Program for Talents in Science and Education of Suzhou (No. KJXW2018079), the clinical Key Disease Diagnosis and Treatment Technology Project of Suzhou (No. LCZX201824, LCZX202003), the Science and Technology Development Plan Project of Changshu (No. CS201819), the Science and Technology Plan Project of Changshu Municipal Health Committee (No. csws201907), the Science and Technology Development Plan (People's livelihood Technology Health applied basic research) of Suzhou (No. SYSD2018063), the Cultivation Research Project of Suzhou Kowloon Hospital (JL201806).

\section{Author contributions}

Ning Li performed conceptualization, methodology, writing-original draft; Xiaoping Li contributed methodology, data curation; Kai Zheng developed software, formal analysis, methodology; Jiaxiang Bai wrote the review \& editing; Weicheng Zhang analyzed data; Houyi Sun and Wei Wang performed formal analysis. Gaoran Ge, Yi Xue, Zhen Wang and Ye Gu performed data curation; Yaozeng $\mathrm{Xu}$ and Jun Zhou performed supervision, conceptualization; Dechun Geng contributed project administration, supervision, writing review \& editing.

\section{Competing Interests}

The authors have declared that no competing interest exists.

\section{References}

1. Adelani MA, Crook K, Barrack RL, Maloney WJ, Clohisy JC. What is the prognosis of revision total hip arthroplasty in patients 55 years and younger? Clin Orthop Relat Res. 2014; 472: 1518-25.
2. Nikolaou VS, Korres D, Lallos S, Mavrogenis A, Lazarettos I, Sourlas I, et al. Cemented Müller straight stem total hip replacement: 18 year survival, clinical and radiological outcomes. World J Orthop. 2013; 4: 303-8.

3. Kurtz SM, Ong KL, Schmier J, Zhao K, Mowat F, Lau E. Primary and revision arthroplasty surgery caseloads in the United States from 1990 to 2004. J Arthroplasty. 2009; 24: 195-203.

4. Bozic KJ, Kamath AF, Ong K, Lau E, Kurtz S, Chan V, et al Comparative Epidemiology of Revision Arthroplasty: Failed THA Poses Greater Clinical and Economic Burdens Than Failed TKA. Clin Orthop Relat Res. 2015; 473: 2131-8.

5. Sadoghi P, Liebensteiner M, Agreiter M, Leithner A, Böhler N, Labek G. Revision surgery after total joint arthroplasty: a complication-based analysis using worldwide arthroplasty registers. J Arthroplasty. 2013; 28: 1329-32.

6. Dorr LD, Wan Z, Shahrdar C, Sirianni L, Boutary M, Yun A Clinical performance of a Durasul highly cross-linked polyethylene acetabular liner for total hip arthroplasty at five years. J Bone Joint Surg Am. 2005; 87: 1816-21.

7. Nich C, Takakubo Y, Pajarinen J, Ainola M, Salem A, Sillat T, et al. Macrophages-Key cells in the response to wear debris from joint replacements. J Biomed Mater Res A. 2013; 101: 3033-45.

8. Schett G, Gravallese E. Bone erosion in rheumatoid arthritis: mechanisms, diagnosis and treatment. Nat Rev Rheumatol. 2012; 8: 656-64.

9. Jacome-Galarza CE, Percin GI, Muller JT, Mass E, Lazarov T, Eitler J, et al. Developmental origin, functional maintenance and genetic rescue of osteoclasts. Nature. 2019; 568: 541-5.

10. Teitelbaum SL. Bone resorption by osteoclasts. Science. 2000; 289: 1504-8.

11. Park JH, Lee NK, Lee SY. Current Understanding of RANK Signaling in Osteoclast Differentiation and Maturation. Mol Cells. 2017; 40: 706-13.

12. Boyle WJ, Simonet WS, Lacey DL. Osteoclast differentiation and activation. Nature. 2003; 423: 337-42.

13. Nelson CA, Warren JT, Wang MWH, Teitelbaum SL, Fremont DH. RANKL employs distinct binding modes to engage RANK and the osteoprotegerin decoy receptor. Structure. 2012; 20: 1971-82.

14. Kobayashi N, Kadono Y, Naito A, Matsumoto K, Yamamoto T, Tanaka S, et al. Segregation of TRAF6-mediated signaling pathways clarifies its role in osteoclastogenesis. EMBO J. 2001; 20: 1271-80.

15. Lin J, Lee D, Choi Y, Lee SY. The scaffold protein RACK1 mediates the RANKL-dependent activation of p38 MAPK in osteoclast precursors. Sci Signal. 2015; 8: ra54.

16. Takayanagi H, Ogasawara K, Hida S, Chiba T, Murata S, Sato K, et al. T-cell-mediated regulation of osteoclastogenesis by signalling cross-talk between RANKL and IFN-gamma. Nature. 2000; 408: 600-5.

17. Asagiri $M$, Sato $K$, Usami $T$, Ochi $S$, Nishina $H$, Yoshida $H$, et al. Autoamplification of NFATc1 expression determines its essential role in bone homeostasis. J Exp Med. 2005; 202: 1261-9.

18. Novack DV. Role of NF-kB in the skeleton. Cell Res. 2011; $21: 169-82$

19. Shi M, Chen L, Xin Z, Wang Y, Wang W, Yan S. Bisphosphonates for the preservation of periprosthetic bone mineral density after total joint arthroplasty: a meta-analysis of 25 randomized controlled trials. Osteoporos Int. 2018; 29: 1525-37.

20. Peichl P, Griesmacher A, Kumpan W, Schedl R, Prosquil E, Bröll H. Clinical outcome of salmon calcitonin nasal spray treatment in postmenopausal women after total hip arthroplasty. Gerontology. 2005; 51: 242-52.

21. Chiu YG, Ritchlin CT. Denosumab: targeting the RANKL pathway to treat rheumatoid arthritis. Expert Opin Biol Ther. 2017; 17: 119-28.

22. Finkel T, Deng C-X, Mostoslavsky R. Recent progress in the biology and physiology of sirtuins. Nature. 2009; 460: 587-91.

23. Houtkooper RH, Pirinen E, Auwerx J. Sirtuins as regulators of metabolism and healthspan. Nat Rev Mol Cell Biol. 2012; 13: 225-38.

24. Someya S, Yu W, Hallows WC, Xu J, Vann JM, Leeuwenburgh C, et al. Sirt3 mediates reduction of oxidative damage and prevention of age-related hearing loss under caloric restriction. Cell. 2010; 143: 802-12.

25. Hirschey MD, Shimazu T, Goetzman E, Jing E, Schwer B, Lombard DB, et al. SIRT3 regulates mitochondrial fatty-acid oxidation by reversible enzyme deacetylation. Nature. 2010; 464: 121-5.

26. Giralt A, Villarroya F. SIRT3, a pivotal actor in mitochondrial functions: metabolism, cell death and aging. Biochem J. 2012; 444.

27. Wu Y-Y, Xiao E, Graves DT. Diabetes mellitus related bone metabolism and periodontal disease. Int J Oral Sci. 2015; 7: 63-72.

28. Lecka-Czernik B. Marrow fat metabolism is linked to the systemic energy metabolism. Bone. 2012; 50: 534-9.

29. Gu W, Chen K, Zhao X, Geng H, Li J, Qin Y, et al. Highly Dispersed Fullerenols Hamper Osteoclast Ruffled Border Formation by Perturbing Ca Bundles. Small. 2018; 14: e1802549.

30. Garbe AI, Roscher A, Schüler C, Lutter A-H, Glösmann M, Bernhardt R, et al. Regulation of bone mass and osteoclast function depend on the F-actin modulator SWAP-70. J Bone Miner Res. 2012; 27: 2085-96.

31. Chellaiah MA, Ma T, Majumdar S. L-plastin phosphorylation regulates the early phase of sealing ring formation by actin bundling process in mouse osteoclasts. Exp Cell Res. 2018; 372: 73-82.

32. Raimondo A, Lembo S, Di Caprio R, Donnarumma G, Monfrecola G, Balato N, et al. Psoriatic cutaneous inflammation promotes human monocyte differentiation into active osteoclasts, facilitating bone damage. Eur J Immunol. 2017; 47: 1062-74. 
33. Ruscitti P, Cipriani P, Carubbi F, Liakouli V, Zazzeroni F, Di Benedetto P, et al. The role of IL-1 $\beta$ in the bone loss during rheumatic diseases. Mediators Inflamm. 2015; 2015: 782382.

34. Gu Y, Wang Z, Shi J, Wang L, Hou Z, Guo X, et al. Titanium particle-induced osteogenic inhibition and bone destruction are mediated by the GSK-3 $\beta / \beta$-catenin signal pathway. Cell Death Dis. 2017; 8: e2878.

35. Gallo J, Goodman SB, Konttinen YT, Raska M. Particle disease: biologic mechanisms of periprosthetic osteolysis in total hip arthroplasty. Innate Immun. 2013; 19: 213-24.

36. Lorenzo J. The many ways of osteoclast activation. J Clin Invest. 2017; 127: 2530-2.

37. Lee K, Seo I, Choi MH, Jeong D. Roles of Mitogen-Activated Protein Kinases in Osteoclast Biology. Int J Mol Sci. 2018; 19.

38. Zhao H, Sun Z, Ma Y, Song R, Yuan Y, Bian J, et al. Antiosteoclastic bone resorption activity of osteoprotegerin via enhanced AKT/mTOR/ULK1mediated autophagic pathway. J Cell Physiol. 2020; 235: 3002-12.

39. Engsig MT, Chen QJ, Vu TH, Pedersen AC, Therkidsen B, Lund LR, et al. Matrix metalloproteinase 9 and vascular endothelial growth factor are essential for osteoclast recruitment into developing long bones. J Cell Biol. 2000; 151: 879-89.

40. Ikeda F, Matsubara T, Tsurukai T, Hata K, Nishimura R, Yoneda T. JNK/c-Jun signaling mediates an anti-apoptotic effect of RANKL in osteoclasts. J Bone Miner Res. 2008; 23: 907-14.

41. Rardin MJ, Newman JC, Held JM, Cusack MP, Sorensen DJ, Li B, et al Label-free quantitative proteomics of the lysine acetylome in mitochondria identifies substrates of SIRT3 in metabolic pathways. Proc Natl Acad Sci USA. 2013; 110: 6601-6.

42. Peng C, Lu Z, Xie Z, Cheng Z, Chen Y, Tan M, et al. The first identification of lysine malonylation substrates and its regulatory enzyme. Mol Cell Proteomics. 2011; 10: M111.012658.

43. Kim H, Lee YD, Kim HJ, Lee ZH, Kim H-H. SOD2 and Sirt3 Control Osteoclastogenesis by Regulating Mitochondrial ROS. J Bone Miner Res. 2017; 32: 397-406.

44. Huh J-E, Shin JH, Jang ES, Park SJ, Park DR, Ko R, et al. Sirtuin 3 (SIRT3) maintains bone homeostasis by regulating AMPK-PGC-1 $\beta$ axis in mice. Sci Rep. 2016; 6: 22511.

45. Ho L, Wang L, Roth TM, Pan Y, Verdin EM, Hsiao EC, et al. Sirtuin-3 Promotes Adipogenesis, Osteoclastogenesis, and Bone Loss in Aging Male Mice. Endocrinology. 2017; 158: 2741-53.

46. Chen G, Deng C, Li Y-P. TGF- $\beta$ and BMP signaling in osteoblast differentiation and bone formation. Int J Biol Sci. 2012; 8: 272-88.

47. Soltanoff CS, Yang S, Chen W, Li Y-P. Signaling networks that control the lineage commitment and differentiation of bone cells. Crit Rev Eukaryot Gene Expr. 2009; 19

48. Croucher PI, McDonald MM, Martin TJ. Bone metastasis: the importance of the neighbourhood. Nat Rev Cancer. 2016; 16: 373-86.

49. Lochner K, Fritsche A, Jonitz A, Hansmann D, Mueller P, Mueller-Hilke B, et al. The potential role of human osteoblasts for periprosthetic osteolysis following exposure to wear particles. Int J Mol Med. 2011; 28: 1055-63.

50. Ding Y, Yang H, Wang Y, Chen J, Ji Z, Sun H. Sirtuin 3 is required for osteogenic differentiation through maintenance of PGC-1a-SOD2-mediated regulation of mitochondrial function. Int J Biol Sci. 2017; 13: 254-64. 